\title{
ANTIMYCOBACTERIAL AND CYTOTOXICITY ACTIVITIES OF FREE AND LIPOSOME-ENCAPSULATED 3-(4'-BROMO[1,1'-BIPHENYL-4-YL)-3-(4-BROMO-PHENYL)-N,N-DIMETHYL-2-PROPEN-1-AMINE
}

\author{
Ana O. de Souza*\# e Célio L. Silva \\ Faculdade de Medicina de Ribeirão Preto, Universidade de São Paulo, Av. dos Bandeirantes, 3900, 14049-900 Ribeirão Preto - SP, Brasil \\ Nelson Durán \\ Instituto de Química, Universidade Estadual de Campinas, CP 6154, 13083-970 Campinas - SP, Brasil \\ Maria H. Andrade-Santana \\ Faculdade de Engenharia Química, Universidade Estadual de Campinas, CP 6066, 13083-970 Campinas - SP, Brasil
}

Recebido em 17/6/09; aceito em 2/12/09; publicado na web em 26/3/10

\begin{abstract}
The antimycobacterial activity of 3-(4'-bromo[1,1'-biphenyl-4-yl)-3-(4-bromo-phenyl)-N,N-dimethyl-2-propen-1-amine (BBAP), free or incorporated in preformed liposomes, on extracellular M. tuberculosis H37Rv was 8 and $25 \mu \mathrm{M}$ (MIC), respectively. Extracellular antimycobacterial activity was not significantly improved by entrapment of BBAP in liposomes, but there was a 6.1-fold reduction of BBAP cytotoxicity on J774 macrophages. Liposomal BBAP or its free form showed $\mathrm{IC}_{50}$ values of 165 and $27 \mu \mathrm{M}$, resulting in a selectivity index $\left(\mathrm{SI}=\mathrm{IC}_{50} \mathrm{MIC}\right)$ of 3.4 and 6.6, respectively. Free BBAP in concentrations from 10 to $80 \mu \mathrm{M}$ were quite effective in eliminating intracellular M. tuberculosis while liposomal formulation was less effective at these concentrations.
\end{abstract}

Keywords: liposome; tuberculosis; 3-(4'-bromo[1,1'-biphenyl-4-yl)-3-(4-bromo-phenyl)-N,N-dimethyl-2-propen-1-amine.

\section{INTRODUCTION}

Tuberculosis still ranks among the world's most deadly infectious diseases, killing around 2-3 million people per year. ${ }^{1}$ A third of the world's population may carry a latent tuberculosis infection, and the lifetime risk of developing tuberculosis ranges between 10 and $20 \% .^{2}$ Although tuberculosis is a preventable and treatable disease, chemotherapy fails to reach its aim due to several reasons that include daily multiple drug administrations for several months, poor patient compliance, drug toxicity and emergence of drug resistance. Thus, one of the current strategies to enhance therapeutic activity, while minimizing toxicity, is to entrap drugs in a delivery system, assuring slow release over extended time periods. Liposome-encapsulated drugs often exhibit reduced toxicity and have also been shown to enhance retention of drugs in tissues, resulting in an improved overall therapeutic efficacy. ${ }^{3}$ Macrophage-specific delivery systems are currently a subject of much interest, since macrophages act as host cells for many parasites and bacteria, which give rise to the outbreak of many human deadly diseases, such as leishmaniasis and tuberculosis.

It has been previously shown that 3-(4'-bromo[1,1'-biphenyl-4yl)-3-(4-bromo-phenyl)-N,N-dimethyl-2-propen-1-amine (BBAP) is a drug with good antimycobacterial activity in vitro ${ }^{4-8}$ but also cytotoxic to mammalian cells. Our group recently prepared inclusion complexes of BBAP in $\beta$-cyclodextrin ${ }^{8,9}$ in order to reduce cytotoxicity and to improve the antimycobacterial effect. Several combined spectroscopy techniques indicated the formation of a complex of $\mathrm{BBAP} / \beta-\mathrm{CD}$ in the molar proportion of 1:1 and 1:2.

Biological assays regarding cytotoxicity towards mammalian cells (J774) and towards a permanent lung fibroblast cell line (V79) indicated no significant toxic effects with the BBAP/ $/$-CD complexes. The complexes were more effective than the free compound on several mycobacteria species. Similar behavior of the inclusion complexes $\mathrm{BBAP} / \beta-\mathrm{CD}(1: 1$ and 1:2) and rifampicin, a front-line antitubercular

*e-mail: olivia@butantan.gov.br

\#Current address: Laboratório de Bioquímica e Biofísica, Instituto Butantan, Av. Vital Brasil, 1500, 05503-900 São Paulo - SP, Brasil drug, was observed for M. tuberculosis H37Rv growing inside J774 macrophages.

These previous results indicate that the entrapment of BBAP is a promising strategy for reduction of cytotoxicity and enhancement of its effectiveness against mycobacteria. In this study, the efficacy of a formulation containing liposome -entrapped BBAP was evaluated in vitro regarding cytotoxicity, extracellular and intracellular antimycobacterial activities.

\section{EXPERIMENTAL}

\section{Preparation of liposomes}

BBAP was synthesized as previously described.${ }^{10}$ Liposomes were composed by phospholipid:cholesterol (60:40 molar), a known composition which provides stability of the liposomal membrane for drug encapsulation. The phospholipid used in this study was the hydrogenated soybean phosphatidylcholine Epikuron 200SH (Lipoid), a commercial soy lecithin useful for liposome preparations due to high percentage of the phosphatidylcholine structural lipids (distearoylphosphatidylcholine (DSPC:0-15\%) and dipalmitoylphosphatidylcholine (DPPC:85-100\%).

Small unilamellar vesicles containing BBAP were prepared by the classical Bangham method as described previously. ${ }^{11}$ Briefly, the lipids (phospholipid: cholesterol 60:40\%) and BBAP were dissolved in chloroform:methanol $(9: 1 \mathrm{v} / \mathrm{v})$ in BBAP/phospholipid ratios of 0 (empty liposomes); $0.1 ; 0.2 ; 0.3 ; 0.4 ; 0.5$; and 0.6 and a dried film was formed by rotatory evaporation at $55^{\circ} \mathrm{C}$. The drug-lipid film was hydrated with $5 \mathrm{~mL}$ of Hepes buffer $(10 \mathrm{mM}, \mathrm{pH}=7.4$ containing $\mathrm{NaCl}$ $120 \mathrm{mM}$ ), forming a $7.5 \mathrm{mM}$ lipid concentration and the suspension sonicated for $30 \mathrm{~min}$ (Ultrasonic Cleaner Unique) to produce small liposomes and maintained in repose during $2 \mathrm{~h}$ and $30 \mathrm{~min}$.

Preformed liposomes were sized by multiple extrusions (15 times) through a drain disk (Millipore C32WP02500) and two stacked polycarbonate membranes (pore sizes $100 \mathrm{~nm}$ - Osmomics K01CP02500) by a high-pressure extruder $\left(10 \mathrm{kgf} / \mathrm{cm}^{2}\right)$ (Lipex Biomembranes, Vancouver, BC). Non-entrapped BBAP was removed 
from the liposome suspension by ultrafiltration through a membrane (YM 10 NMWL 10.000 - Amicon 13622) in an Amicon cell (Model $850)$ under nitrogen pressure $\left(2.5 \mathrm{kgf} / \mathrm{cm}^{2}\right)$.

\section{Characterization of liposomes}

Phospholipid content in liposomes was characterized by quantification of total phosphate in samples, according to the methodology developed by Chen et al.. ${ }^{12}$

To measure BBAP concentration, the liposomes were lysed with ethanol and the absorbance of the solution measured spectrophotometrically at $266 \mathrm{~nm}$ (Spectrophotometer, Hitachi U-200). BBAP concentrations in the liposomes were determined by comparison with a standard curve prepared with BBAP from 0 to $20 \mu \mathrm{g} / \mathrm{mL}$ and the trapping efficiency was calculated as follows:

$\% \mathrm{E}=100 *(\mathrm{mols} \mathrm{BBAP} / \mathrm{mols} \text { lipids })_{\text {final }} /(\text { mols BBAP } / \text { mols lipids })_{\text {initial }}$

$\% \mathrm{E}=$ Percent of encapsulation

Liposome mean diameter and particle size distribution were determined by quasi elastic light scattering at $90^{\circ}$ with a He-Ne laser, $633 \mathrm{~nm}$ and at $25^{\circ} \mathrm{C}$ (QLS - Light Scatter - Malvern Instruments Autosizer model 4700, UK).

\section{Biological assays}

\section{Bacteria}

M. tuberculosis H37Rv ATCC 27294, was grown in LoweinsteinJensen medium at $37^{\circ} \mathrm{C}$ for 3 weeks and subcultured in Middlebrook $7 \mathrm{H} 9$ broth medium, supplemented with OADC at $37{ }^{\circ} \mathrm{C}$ for $10 \mathrm{~d}$. Cell concentration in the mycobacterial suspension was adjusted by optical absorbance in comparison with a standard curve and diluted in Middlebrook $7 \mathrm{H} 9$ broth medium to $4.0 \times 10^{5}$ mycobacteria/mL to determine the minimal inhibitory concentration (MIC) or in RPMI $1640\left(5.0 \times 10^{6}\right.$ mycobacteria/mL) to detect macrophage infection in intracellular antimycobacterial activity. ${ }^{13}$

\section{J774 Macrophages}

J774 cells were grown as monolayers in RPMI 1640 medium supplemented with $10 \%$ heat inactivated fetal calf serum (FCS), $100 \mathrm{IU} / \mathrm{mL}$ penicillin and $100 \mu \mathrm{g} / \mathrm{mL}$ streptomycin in a humidified incubator with a $5 \% \mathrm{CO}_{2}$ atmosphere at $37{ }^{\circ} \mathrm{C}$.

\section{Extracellular antimicrobial susceptibility testing - minimal inhibitory concentration (MIC)}

Free BBAP dissolved in dimethylsulphoxide (DMSO), empty and BBAP-containing liposomes were sterilized by passage through a $0.22 \mu \mathrm{m}$ PFTE filter and diluted to the range of 10 to $160 \mu \mathrm{M}$ in Middlebrook 7H9. Rifampicin was dissolved in DMSO, diluted in Middlebrook $7 \mathrm{H} 9$ broth and used as a reference drug at $12 \mu \mathrm{M}$.

Tests were performed by the microplate Alamar Blue assay, as previously described. ${ }^{14}$ M. tuberculosis $\mathrm{H} 37 \mathrm{Rv}(100 \mu \mathrm{L})$ were seeded in a 96-well microplate $\left(4.0 \times 10^{5}\right.$ mycobacteria $\left./ \mathrm{mL}\right)$ containing $100 \mu \mathrm{L}$ of serial dilutions of BBAP - entrapped liposomes, empty liposomes or free BBAP. Plates were incubated at $37^{\circ} \mathrm{C}$ for $6 \mathrm{~d}$, followed by the addition of $25 \mu \mathrm{L}$ mixed(1/1) Alamar Blue reagent and $10 \%$ Tween $80(\mathrm{v} / \mathrm{v})$ to each well. Plates were reincubated at $37^{\circ} \mathrm{C}$ and after $24 \mathrm{~h}$, a change in color from blue to pink, indicated mycobacterial growth.

Control wells consisted of either bacterium only, drugs with medium or only medium. The visual MICs were defined as the lowest drug concentration that prevented a color change from blue to pink.

\section{Cytotoxicity to mammalian cells - J774 macrophages}

The BBAP cytotoxic effect was assayed on J774 macrophages by measuring the reduction of 3-(4,5-dimethylthiazole-2-yl)-2,5diphenyl tetrazolium bromide (MTT) according to Denizot and Lang ${ }^{15}$ and expressed as $\mathrm{IC}_{50}$ values (concentration in which at least $50 \%$ of the cells are viable). Stock solutions of BBAP were prepared in DMSO and diluted in RPMI 1640 without phenol red. The final solvent concentrations in the assay were less than $0.3 \%$ and each sample concentration was tested in six replicates, and repeated three times in separate experiments. Cells $\left(2.0 \times 10^{6}\right.$ cells $\left./ \mathrm{mL}\right)$ added to 96-well plates had the medium removed after $12 \mathrm{~h}$ and replaced by a medium containing free or liposomal BBAP in concentrations ranging from 10 to $160 \mu \mathrm{M}$. As controls, macrophages were treated with empty liposomes and with RPMI 1640, and considered as $100 \%$ viable. Cells were exposed for $24 \mathrm{~h}$ to test medium with or without drugs (controls).

After exposure to the test compounds, the medium in the wells was removed and replaced by $0.1 \mathrm{~mL}$ of serum-free medium containing MTT $(0.5 \mathrm{mg} / \mathrm{mL})$. Following $4 \mathrm{~h}$ incubation, the supernatant was removed and the blue formazan product obtained was dissolved in $0.1 \mathrm{~mL}$ of isopropanol in $0.1 \mathrm{M} \mathrm{HCl}$. Plates were stirred for 15 min on a microtiter plate shaker and absorbance was read at $570 \mathrm{~nm}$.

\section{Intracellular antimicrobial susceptibility testing}

J774 macrophages were harvested and plated at a concentration of $5.0 \times 10^{5}$ cells per well in 24-well tissue culture plates. After overnight incubation, the cells were overlaid with $1 \mathrm{~mL}$ of a suspension of M. tuberculosis H37Rv (5.0x $10^{6}$ mycobacteria/mL) adjusted to yield a multiplicity of infection of 10 mycobacteria per macrophage. Cells were infected for 3-4 h and washed with phosphate-buffered saline (PBS), $\mathrm{pH}=7.4$, to eliminate unbound mycobacteria. M. tuberculosis $\mathrm{H} 37 \mathrm{Rv}$-infected cells were fed again with culture medium containing free or liposomal BBAP in concentrations ranging from 10 to $160 \mu \mathrm{M}^{8,13}$

Control experiments included, RPMI 1640 medium, empty liposomes and rifampicin at $12 \mu \mathrm{M}$. Rifampicin and the empty liposomes were administered diluted in Middlebrook 7H9 broth. After 72 h, cells were washed with PBS to eliminate extracellular bacteria and lysed by addition of $500 \mu \mathrm{L}$ of $0.25 \%$ (wt/vol) sodium dodecyl-sulphate in phosphate-buffered saline (PBS - $\mathrm{pH}=7.4$ ). The lysates were serially diluted and $100 \mu \mathrm{L}$ aliquots were dispersed onto $7 \mathrm{H} 10$ agar plates. The colony formation units (CFU) of M. tuberculosis H37Rv were counted 2-4 weeks after incubation at $37{ }^{\circ} \mathrm{C}$ with $5 \% \mathrm{CO}_{2}$.

\section{Selectivity index}

Selectivity index (SI) was calculated for free and liposomal BBAP formulations taking into account the MIC against $M$. tuberculosis $\mathrm{H} 37 \mathrm{Rv}$ and the $\mathrm{IC}_{50}$ on J774 cells ( $\left.\mathrm{SI}=\mathrm{IC}_{50} / \mathrm{MIC}\right)$ by the MTT assay. ${ }^{8}$

\section{Statistical analysis}

Results of cytotoxicity assays were expressed as cellular viability and displayed graphically using the computer software package Origin-Data Analysis and Technical Graphics, version 6.0 (Copyright Software, Inc.). The $\mathrm{IC}_{50}$ values, concentrations that produced a $50 \%$ inhibitory effect on the evaluated parameter, were obtained by data interpolation.

Statistical analysis of the intracellular antimycobacterial activity was performed by the graph Prisma Software. 


\section{RESULTS AND DISCUSSION}

\section{Liposomes characterization}

Figure 1A shows the profile relating the initial and final drug/lipid ratios. The BBAP encapsulation increased from 0.1 to 0.3 , remaining constant at higher drug/lipid ratios. These results indicate the saturation of the lipid bilayer, from which the encapsulation efficiencies decreased strongly (Figure 1B). The final drug/lipid ratios were 10 times lesser than the initial ones, characterizing a low capability of the lipid matrix for BBAP entrapment. Further optimization of the formulation in terms of phospholipids and cholesterol concentration could enhance the packing of lipids in the matrix, providing higher entrapment efficiencies.
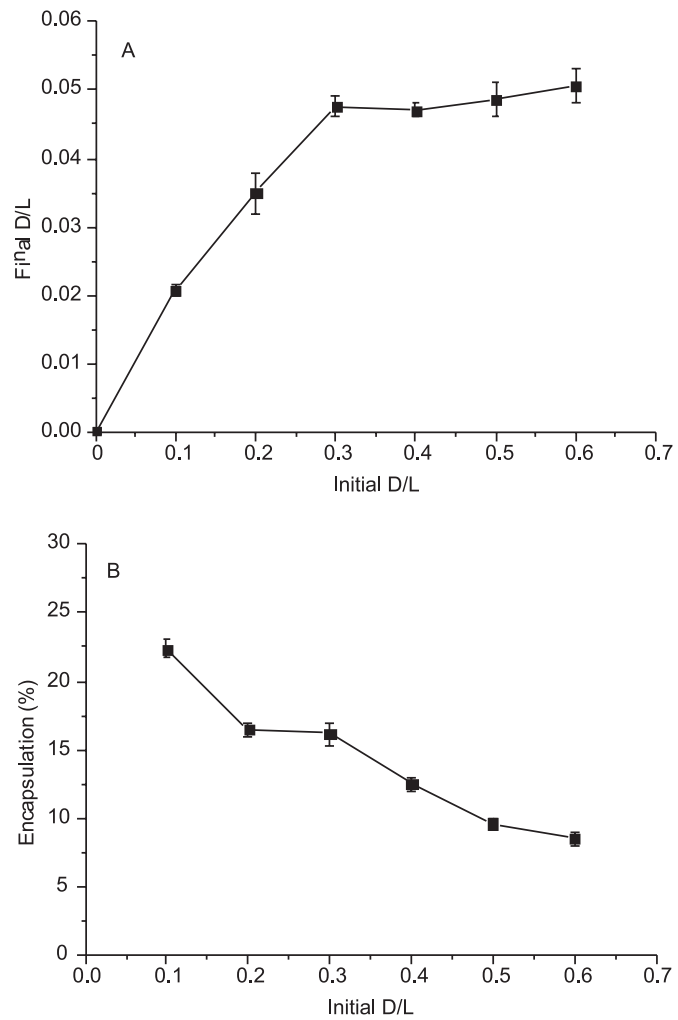

Figure 1. Profile relating the initial and final drug/lipid ratios (A) and encapsulation efficiencies of BBAP in liposomes $(B) . D / L=$ drug/lipid ratios. Bars represent mean $\pm S E M$ of two independent experiments

The average diameter of liposomes sizes was $130 \mathrm{~nm}$ and the polydispersity index was 0.3 . The distributions were monodisperse in the range of 100 to $150 \mathrm{~nm}$.

\section{Biological assays}

Antimycobacterial activity of free BBAP and of liposomes carrying BBAP was assayed on extracellular M. tuberculosis $\mathrm{H} 37 \mathrm{Rv}$ and the MIC values obtained were, respectively, 8 and $25 \mu \mathrm{M}$ (Table 1).

As shown in Figure 2 liposomes entrapment of BBAP successfully decreased the cytotoxicity of BBAP. The $\mathrm{IC}_{50}$ of liposomes with free and entrapped BBAP were 27 and $165 \mu \mathrm{M}$, respectively. The cytotoxicity of the compound was reduced by 6.1 fold, showing that this pharmaceutical formulation was important in reducing the toxicity of the drug, although the increase of the extracellular antimycobacterial activity was modest. Empty liposomes were not toxic to the cells (Figure 2). Table 1 shows
Table 1. Antimycobacterial parameters of free and liposome-entrapped BBAP

\begin{tabular}{lccc}
\hline Samples & $\mathrm{MIC}^{*}(\mu \mathrm{M})$ & $\mathrm{IC}_{50}^{* *}(\mu \mathrm{M})$ & $\mathrm{SI}=\mathrm{IC}_{50} / \mathrm{MIC}$ \\
\hline Free BBAP & 8 & 27 & 3.4 \\
Liposomal BBAP & 25 & 165 & 6.6 \\
\hline
\end{tabular}

$* \mathrm{MIC}=$ corresponds to the MIC against $M$. tuberculosis $\mathrm{H} 37 \mathrm{Rv}$; $* * \mathrm{IC}_{50}=$ corresponds to the $\mathrm{IC}_{50}$ on $\mathrm{J} 774$ macrophages by the MTT assay; $\mathrm{SI}=\mathrm{IC}_{50} / \mathrm{MIC}$.

the selectivity indexes $\left(\mathrm{SI}=\mathrm{IC}_{50} / \mathrm{MIC}\right.$ ) of free BBAP and BBAP entrapped in liposomes and the values are 3.4 and 6.6, respectively. For a drug to be considered a good candidate against tuberculosis its SI should be higher than 10.

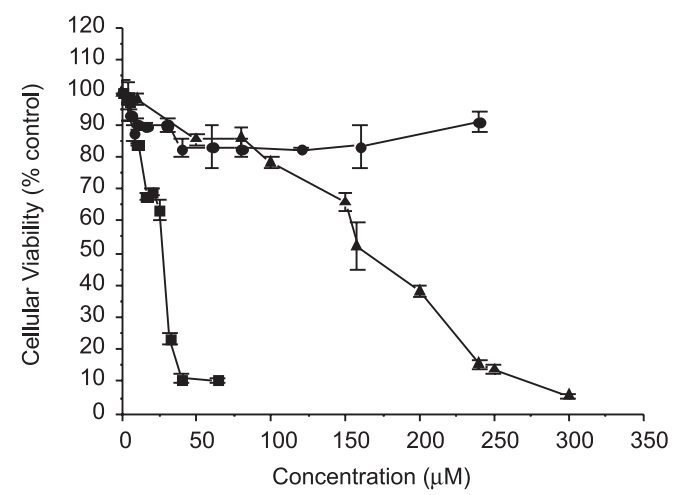

Figure 2. Cytotoxicity to J774 macrophages of free BBAP(ם), empty liposomes $(\bullet)$ and liposome-entrapped BBAP $(\mathbf{\Delta})$ determined by the MTT assay. Bars represent mean \pm SEM of three independent experiments

Although lower efficacy of liposomal BBAP compared to free BBAP, these results indicate that BBAP liposomal formulation is promising for antimycobacterial activity as demonstrated by decreasing of BBAP cytotoxicity and the selectivity index. However, an optimization of liposome composition, drug encapsulation and release as well as in vitro and in vivo studies are required for a complete evaluation of antimycobacterial efficacy of liposomal BBAP.

Free BBAP in concentrations ranging from 10 to $80 \mu \mathrm{M}$ was significantly effective in eliminating intracellular $M$. tuberculosis H37Rv while BBAP liposomal formulation was not so efficient at the same concentrations (Figure 3 ) in comparison to control cells, which were infected and treated only with culture medium or empty liposomes (data not shown). The effects of the BBAP liposomal formulation on the biological results could be related to the slow release of drug and liposome phagocytosis. Enhancements on these

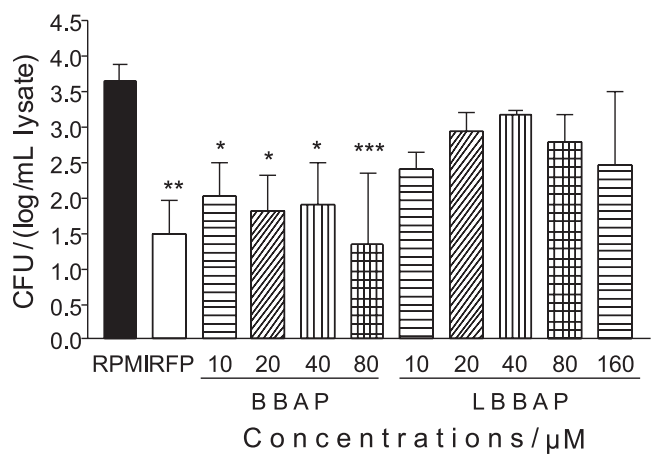

Figure 3. Effect of free and liposomal BBAP, and rifampicin (RFP) against M. tuberculosis H37Rv growth inside $J 774$ macrophages. Macrophages were lysed to determine CFU of M. tuberculosis H37Rv following the procedure described in the text. Bars represent mean $\pm S E M$ of three independent experiments. RPMI 1640 and rifampicin $(R F P-12 \mu M)$ were used as controls 
aspects could be provided by adding lipids of low phase transition temperature and cationic lipids to the formulation.

As expected rifampicin at $12 \mu \mathrm{M}$ was effective and eliminated more than $2 \log$ in the bacillus number.

\section{CONCLUSION}

The encapsulation of BBAP in liposomes and the in vitro evaluations of the cytotoxicity, extracellular and intracellular antimycobacterial activities were characterized. The liposomal formulation is promising for the treatment of $M$. tuberculosis infections due to its reduced citotoxicity and higher selectivity index, compared to free BBAP.

\section{ACKNOWLEDGMENTS}

The authors thank Conselho Nacional de Desenvolvimento Científico e Tecnológico (CNPq) for financial support to A. O. de Souza (Profix-CNPq) and Brazilian Tuberculosis Research Network (REDE TB - Instituto do Milênio).

\section{REFERENCES}

1. Corbett, E. L.; Watt, C. J.; Walker, N.; Mayer, D. B. M.; Willians, B. G.; Raviglione, M. C.; Dye, C.; Arch. Intern. Med. 2003, 163, 1009.

2. Vynnycky, E.; Fine, P. E.; Epidemiol. Infec. 1997, 119, 183.

3. Pandey, R.; Khuller, G. K.; J. Antimicrob. Chemother. 2005, 55, 430.
4. De Souza, A. O.; Aily, D. C. G.; Sato, D. N.; Durán, N.; J. Antimicrob. Chemother. 1998, 42, 407.

5. De Souza, A. O.; Junior, R. R. S.; Ferreira-Julio, J. F.; Rodriguez, J. A.; Melo, P. S.; Haun, M.; Sato, D. N.; Durán, N.; Eur. J. Med. Chem. 2001, 36,843 .

6. De Souza, A. O.; Pereira, D. G.; Durán, N.; Ann. Rev. Biomed. Sci. 2002, 4,53 .

7. De Souza, A. O.; Hemerly, F. P.; Busollo, A. C.; Melo, P. S.; Machado, G. M. C.; Miranda, C. C.; Santa-Rita, R. M.; Haun, M.; Leon, L. L.; Sato, D. N.; De Castro, S. L.; Durán, N.; J. Antimicrob. Chemother. 2002, 50, 629.

8. De Souza, A. O.; Santos, R. R.; Sato, D. N.; De Azevedo, M. M. M.; Ferreira, D. A.; Melo, P. S.; Haun, M.; Silva, C. L.; Durán, N.; J. Braz. Chem. Soc. 2004, 15, 682.

9. De Souza, A. O.; Alderete, J. B.; Faljoni-Alario, A.; Silva, C. L.; Durán, N.; J. Chil. Chem. Soc. 2005, 50, 591.

10. De Conti, R.; Gimenez, S. M. M.; Haun, M.; Pilli, R. A.; De Castro, S. L.; Durán, N.; Eur. J. Med. Chem. 1996, 31, 915.

11. Bangham, A. D.; Standish, M. M.; Watkins, J. C.; J. Mol. Biol. 1965, 13, 238. 12. Chen, P. S.; Toribara, T. Y.; Warner, H.; Anal. Chem. 1956, 28 , 1756.

13. Oh, Y. K.; Nix, D. E.; Straubinger, R. M.; Antimicrob. Agents Chemother. 1995, 39, 2104.

14. Collins, L. A.; Franzblau, S. G.; Antimicrob. Agents Chemother. 1997, 41,1004

15. Denizot, F.; Lang, R.; J. Immun. Methods 1986, 89, 271. 